\section{LE CORBUSIER: CONCURSOS Y PALACIOS}

\section{CORE}

Fernando Zaparaín Hernández
RESUMEN Cuando Le Corbusier y a había pasado de los cuarenta años se enfrentó a dos concursos internacionales: el Palacio de pos de estado con Ginbra (1926-31) ye I Palacio de los Soviets en Moscú (1931-32) con los que intentaba acceder a grandes encarcasos estuvo entre los vencedores pero por conveniencia e indecisión, los poderes politicos optaron por soluciones academicistas. Al conocer las decisiones definitivas, Le Corbusier y todos sus contactos del Movimiento Moderno orquestaron diversas polémicas ante los medios de comunicación y los gobernantes por entender que el progreso se veial lastrado por el triunto de propuestas reac-

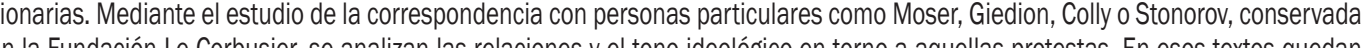
reflejados los principales rassos que caracterizan la modernidad como su internacionalización, I I seguridad en la propia misión, Ia

defensa de lo nuevo o el método dialéctico.

SUMMARY When Le Corbusier had already passed the age of forty he confronted two international competitions: the Palais des Nations in Geneva (1926-1931) and the Palace of the Soviets in Moscow (1931-32), with which he attempted to access large was among the winners, but through convenience and indecision the political powers opted for solutions from academicians. Upon knowing the final decisions, Le Corbusier and all his contacts in the Modern Movement orchestrated several controversies with the media, and the people in power, to convey that progress seemed burdened by the triumph of reactionary proposals. The relationsColly or Stonorov, which are preserved in the Le Corbusier Foundation. These texts reflect the main features that characterize modernity such as its internationalization, the security in the mission itself, the defence of the new or the dialectical method. KEY WORDS Le Corbusier; Palais des Nations; Palace of the Soviets; competitions; correspondence; modernity.

Personade contacto/Correspondingauthor:Zzaparain@gmail..com. Escuela Técnica Superiorde Arquitectura. UniversidadddeValladolid. e Corbusier cumplió cuarenta años en 1927'. En lugar en la cultura arquitectónica moderna gracias a su actividad en diversas facetas. Para los primeros años de la década de los treinta se había propuestollegar a una mayor escala de intervencíńn, que solo consiguro en parte, como demuestran estas palabras de su Obra

"Qué hemos hecho por tanto durante estos años 1929/34? En primer lugar, algunos edificios y después grandes estudios de urbanismo. Estos edificios han jigado el papel de laboratorios. Hemos querido que cada elemento construido durante esos años fuera el avance experimental que permitiera tomar con toda seguridad las iniciativas indispensables en urbanismo".

Efectivamente, un sencillo vistazo al catálogo de su obra deja claro que no consiguió materializar todo el potencial que había acumulado Sus realizaciones siguie ron limitándose a sofisticadas villas para la élite de su cículo próximo de admiradores intelectuales, cuando no de su entorno familiar. Hubo también algunos edificios

colectivos e institucionales, pero la historia vetó su acceso a encargos públicos de envergadura. Sólo al final de pestadisas llamarían a su puerta.

tivo fracaso fueron dos convocatorias internacionales, el único ámbito donde podrí haber conseguido la repercusión que cré́ merecer Se trató de los concursos para el Palacio de las Naciones (1926-31) y el Palacio de los Soviets (1931-32). En ambos estuvo muy cerca de ser elegido, pero los poderes en los que confiaba para triunfar optaron por soluciones convencionales. Aunque no pudo construir sus propuestas aprovechó para convertir la publicación de los resultados en una batalla por la modernidad. A ello contribuyó poderosamente la movilización de relaciones que orquestó entre sus seguidores y en la opinión pública. De esa forma la historia de la arquitectura se hizo eco de la potencia de sus ideas aunque no se construyeran. Lo que permaneció fue la polémica, uno de los elementos constitutivos de la vanguardia, que se justifica precisamente como lo nuevo y que para ello necesita un enemigo conservador

1. Sobre el carácter de encrucujada cultura de este año vease Lahuerta, Juan Jose: 1927. La abstracción necesaria en el arte y la arquitectura europeos de entreguerras. Barcelona: Anthropos, 1989

2. Boesiger, Willy; Girsberger, Hans: (Ed.): Le Corbusier et Pierre Jeanneret, Oeuure complète 1929-34 vol. 2. Zurich: Editions Girsberger, 1934, p. 15 

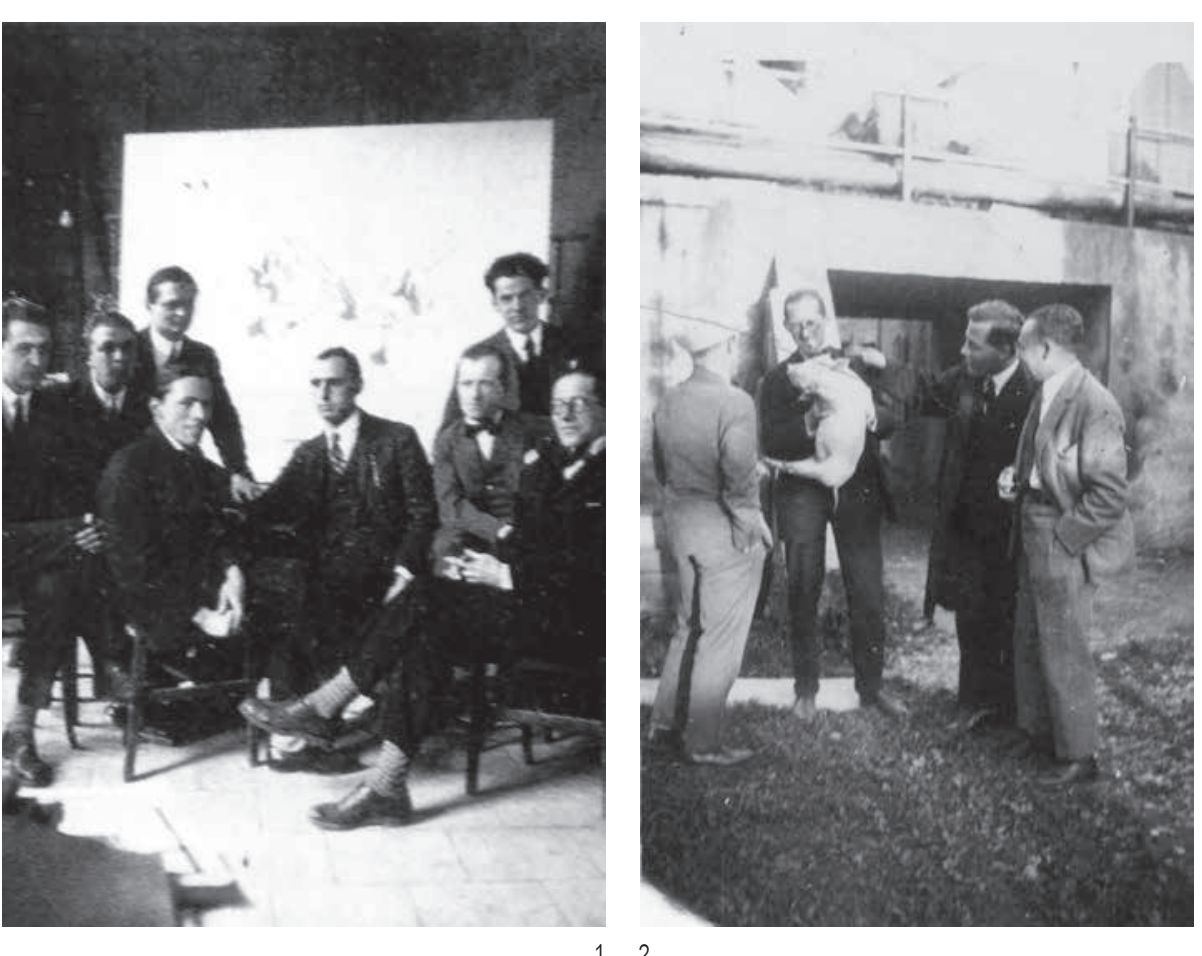

al que enfrentarse heroicamente (igura 1), que aquí se personificó en el academicismo

Este artículo se limitará a la correspondencia privada de Le Corbusier en tomo a ambos concursos, especialStonoror No nos detendrem más que lo improscindible en las incichcies del proceso las relaciones con he sido suficientemente estudiados? Ahora interesa más bien conocer cómo funcionaron las conexiones persohien cos las con disputadas convocatoriast. Podemos adelantar aue correo personal coincide con la linea reivindicativa de sus requerimientos institucionales, pero añade datos sobre la trama subterránea de apoyos y aporta uces sobre la consolidación de relaciones ideológicas con varias figuras junto a las que formarí el frente común de los CIAM y el CIRPAC.

EL PALACIO DE LAS NACIONES (1926-31) Como el propio Le Corbusier explica en la Obra Complea, su proyecto, "...fue designado para el primer premio y curso internacional de 1927, en el que 377 proyectos recibieron descle todas las partes del mundo, represenTan na de menos que $12 \mathrm{~km}$ de planos Maniobras, de as cuales 10 menos que puede decirse es que estaban desprovistas de toda honestidad, arancaron a los auban tión del palacio se confió a el fruto de su trabajo, y la ejecución del palacio se confió a

Tenzó en la fase misma de condaso, an es de conocer el fallo del jurado. Durante la rede Le Corburer sobre las difioultades que encontrarín entre los burócratas. Así parece corroborarlo una anécdota de tono muy beligerante, transmitida por Alfred Roth

3. La bibliografía sobre ambos concursos se recoge al final del articulo. Es muy amplia y aquí se mencionará la fundamental porque este artículo no es reco. pilatorio y se centra en la correspondencia particular corbuseriana. En los estudios monográríicos se ha publicado sobre todo la correspondencia oficial, per aparecen menos referencias a las cartas personales de Le Corbusier con otros colegas. Practicamente ninguna se ha traducido al castellano. Además, aunque algunas son citadas, no siempres se han reproducido. Hasta ahora esas cartas se han usado mas bien para fijar fechas y datos. Los textos, en si mismos se

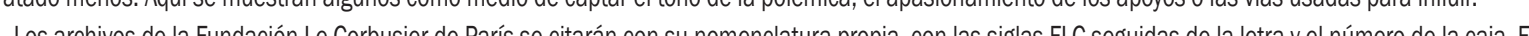
4. Los archivos del la fundachn Le corbusier de Parts 5. Boesiger, Willy; Stonorov, Oscar. (Ed.): Le Corbusier et Pierre Jeanneret, Oeuvre complète 1910-29 vol. 1. Zurich: Editions Girsberger, 1937 (edición en francés), p. 160 6. Alfred Roth (1903-1998) era suizo, estudió en París y colaboró en el estudio de Le Corbusier durante el año 1927. A partir de 1956 fue profesor de la ETh
de Zurich. Para una biografía suya ver Midant, Jean-Paul. (dir): Diccionario Akal de la Arquitectura del Sigílo XX. Madrid: Ediciones Akal, 2004, p. 785.
Le Corbusier, después de haber posado con el equipo que le ayudó en el concurso, se hizo otra foto en la que aparece sujetando un cerdito (figura 2). Después rehusó lavarse las manos comentando, "no es necesario: vengo de Ginebra, donde he estrechado tantas manos de diplomáticos que estaban más sucias

En esta anécdota se atisba cómo los concursos corbuserianos tenian varios frentes. Alrededor de la propuesformal hay tambien una labor iconica, de gestos y luch fente al poder, del que paradójicamente se depende y que se acabaría cortejando. Una fologralla teónicamen(te entranable se convierte en un manifiesto al añadirle lase del maestro luchador. Por otra parte, la mención a los diplomálicos cuyas manos se han estrechado da a entender que Le Corbusier hizo gestiones durante los meses de redacción de su propuesta, en la segunda tad de 1926. Por ejemplo, sabemos que en una carta de 30-11-26 se dirigio a Karl Moser (1860-1931), represenlante suizo en el jurado, para decirle que en una visita a slar habla deteclado dudas sobre si realmente este sería el emplazamiento del proyecto. Admitia que su pregunta gual no era licita y tambien mencionaba que era su intención haber visitado a Huston y Lloyd, ambos funcionario de la Sociedad de Naciones (S.D.N.) involucrados en el concurso.

La conexión con Moser va a ser una constante a lo argo de todo el proceso, pasando desinhibidamente po encima de su condición de jurado. Aunque en aquella epoca ya tenía 66 años y sus realizaciones personales no estaban en la vanguarda, desde su tarea docente en la ETH de Zurich impulsó la arquitectura moderna en

Suiza y aglutinó el ala progresista del concurso $0^{9}$. Las re laciones de los dos compatriotas están documentadas, hasta donde conozco, desde una carta del 17-6-1919". Moser estaba propuesto para el jurado desde finales de diciembre de 1924, cuando seis paises fueron invitados mandar sus candidatos. Desde aquel primer contacto las vinculaciones continuaron y el 21 de mayo de 1926 este profesor escribe a Le Corbusier desde Zurich para invitarle a una conferencia"1", casi coincidiendo con el esablecimiento del programa definitivo del concurso (17 de abril de ese año) que estaba siendo difundido durante $e$ mes de mayo por todos los paises. Es interesante la devoción que este miembro del jurado y profesor consagrado manifiesta a Le Corbusier, mucho más joven que ể. Señor y muy apreciado colega. Desde hace algunos años sigo con el interés más grande sus esfuerzos para un desarrollo contemporáneo de la arquitectura. Las verdades que pronuncia en sus libros me impresionan tanto como sus obras realizadas que veo de tiempo en tiempo publicadas en los periódicos franceses

La sintonia se mantuvo a lo largo del proceso. Es comer candidato de Moser fue Le Corbusier. Entre las innumerables gestiones y cartas oficiales ya publicadas sobre su apoyo, podemos destacar la derensa que en mayo de 1927 hace Karl Moser ante numerosos funcionarios de la Sociedad de Naciones y ante el jefe del Departamento de trabajos publicos de Ginebra

Este compromiso tan denso entre los dos personaer se manturoy cuando Le Corbusier inpulsa la fundación de los CIAM, con el congreso inicial de La Sarraz de 8. FLC E2(16) 367. También se conoce por la copia del Archivo de la S.D.N. 37/39306/28594, citada en Rovira, Josep Maria: "Le Corbusier en el concurso de

. Sobre la actuación de Moser en el jurado ver Strebel, Enst. "Le déroulement des séances dujury d'aprés les notes de Karl Moser le representante suisse." .

10. FLCE2(16) 365.

12. FLC E2(16) 366. "Monsieur et très apprecié confrère. Depuis quelques années je poursuivis avec le plus grand intérêt vos efforts pour un développement contemporain de l'architecture. Les verités que vous prononcez dans vos lives m'impressionnent autant que vos oeuvres éxecutés que je vois de temps en temps publiées dans les journaux françals. 13. Godoli, Ezi: "II Palazzz della Societé delle Nazioni: dal concurso alla costruzione. Cronaca e documenti" en Anzivino, Ciro; Godoli, Ezio: Ginevra 1927: 


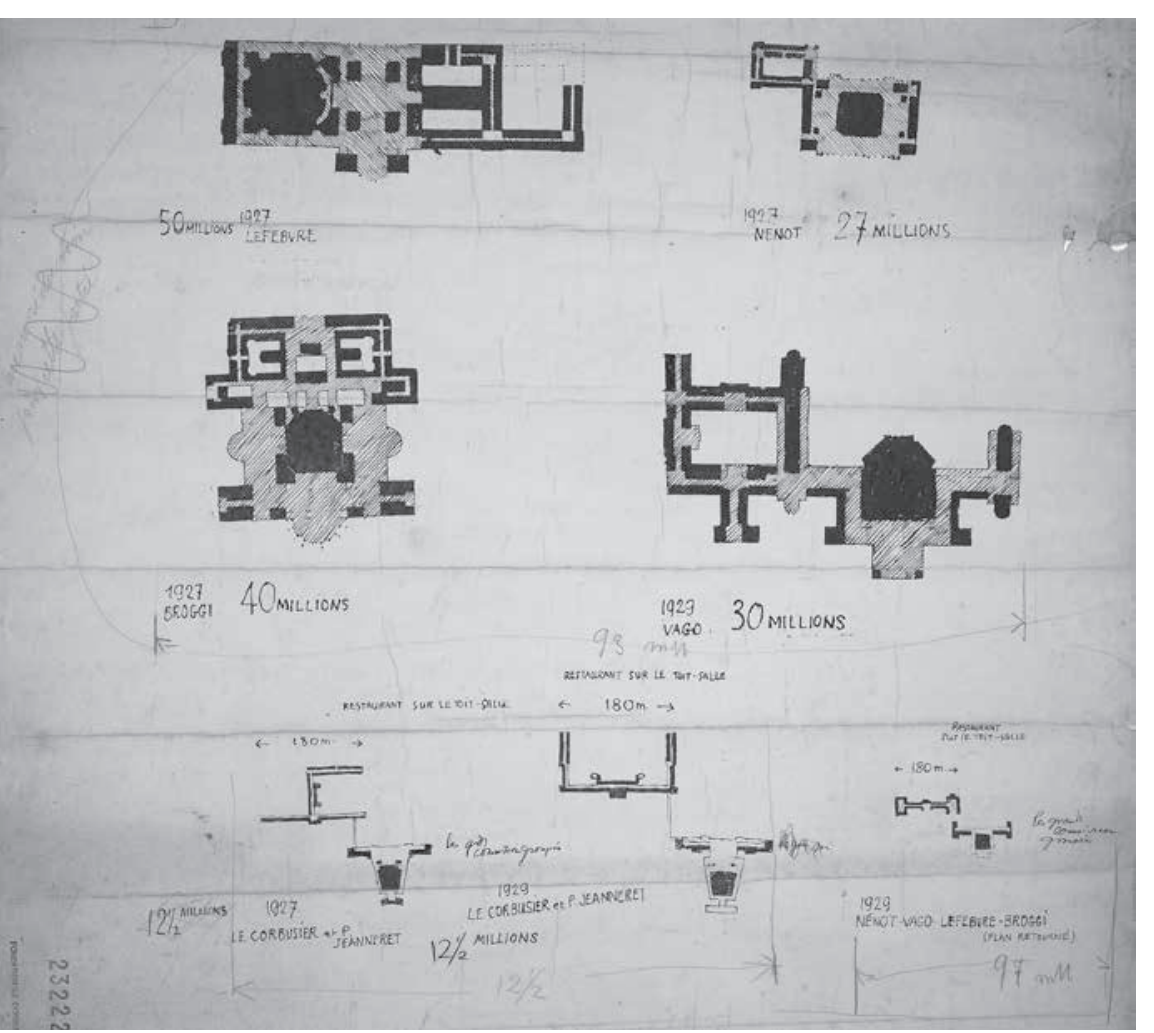

1928, Moser se convierte en presidente. Allí encontramos lambién a otros viejos conocidos como Meyer, tercer arquitecto propuesto por Moser en el concurso, y por supuesto, Sigfried Giedion, con los que Le Corbusier había consolidado sus lazos precisamente en medio de la poémica del Palacio de Ginebra. Se corrobora asi que los CIAM surgen en la agitacion producida por el concurso de Ginebra, que abrió los ojos a los más comprometidos sobre la necesidad de aunar fuerzas a traves de un instrumento de difusion. El concurso y su correspondiente polémica crearon bandos muy definidos que obligaron a posicionarse e hicieron avanzar la conciencia moderna. Una vez conocido el extraño veredicto del jurado que dejaba desierto el primer premio y repartía tres series de nueve ex-aequo, se puso en marcha una cadena de reacciones que tiene a Le Corbusier por centro. Muy pronto entrará en escena olro personaje clave de la modernidad: el tambièn profesor de la universidad de Zurich, suizo y casi coetaneo, Sigfried Giedion (1888-1968). Las relaciones entre ambos se fueron estrechando hasta llegar a la

undación de los CIAM en 1928, en los que Giedion fue nombrado primer secretario general. La carta más antigua entre ellos que conozco no tiene fecha pero lleva un matasellos de LExposition internationale des arts decoratifs et industriels modemes que permite datarla entre abrily octubre de $1925^{\circ}$. En 1926 Giedion le solicita unas fotografías de su obra a Le Corbusier', lo que indica que ya le está inclyendo en sul labor críticay divulgativa de la modernidad. La primera carta entre ellos que menciona el concurso es del 14 de abril de 1927'16. En ella Giedion contesta a otras recibidas de Le Corbusier. Despacha con el de diversas cuestiones y se expresa con un tono devoto. Todo ello sugiere que su sintonía iba más allá de las peripecias de esta competición.

"Usted sabe bien cómo le estimo. Sobre todo su fantasía en arquitectura y la facilidad y la fuerza con que arroja a lanza hacia el futuro, recogiendo también el pasado"17. Giedion explica el tratamiento que recibe la arquicha clara su militancia a favor de lo que ya denomina como

14. FLC A2(15). En la Fundación esta carta todavía no ha sidd incluida en el dossier sobre Sigfried Giedion, E2(03) 187- 246, que comienza en 1927. 15. FLCT1(1) 25

16. FLC E2(03) 96 a 98. Tanto Godoli como Quincerot, en sus diversas publicaciones mencionadas en la bibliografía, pasan por alto esta carta, que comple17.

17.FLCE2(03) 96. "Vous savez bien con
Movimiento Moderno. Para ello establece un enemigo, que designa hasta cuatro veces con un término político Ia mente de lasticas que hace la vanguardia (en Holanda, por ejemplo); las observaciones de

(a) compromiso,

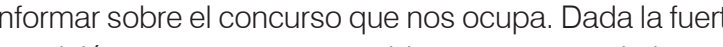
vosicón, pare cestar asarse que an ara de la nuepuede confiar solo en las propuestas arquitectincas y (

Lo que le interesa seguramente antes que nada: las 'negociaciones' de Ginebra. Estamos aquí se puede imadecir nada definitivo berona comunicación. No se puede común del grupo nórdico: el italiano y el español tienen aspecto de no haber visto nunca un proyecto moderno. Es justo la reacción la que parece desorientada"19.

Todo este cruce de información se inserta y justifica en una lógica de grupos enfrentados. Desde luego, hay uno muy especial formado por los tres suizos, pero dentro del jurado se establecen otros, como el "nórdico" o el de "Ia reacción". En otro lugar de la carta, Giedion se refiere a todos como los "vejetes" del jurado, y efectivamente lo eran respecto a él (39 años) y Le Corbusier (40). Se supone que el grupo "nórdico", además del suizo Moser (66) incluiría al holandés Berlage (71), al inglés John J. Burnet al austríaco Josef Hoffmann (57), al sueco Ivar Tengbom y al belga Victor Horta (66). Por muy nórdicos que fueran sabemos que no tuvieron unidad de criterio respecto a lo

moderno. Por ejemplo, Berlage se resistió a reconocer proyecto corbuseriano, pero se fue inclinando cada vez más hacia ISs modenos, hasta el punto de integrarse min el peculiar desglose de Giedion, fuera español Carlos Gato (52), el italiano Attilio Mugia (67) y el francés Charles Lemeresuier (57)20. En seguida Giedion pasa revista a las actitudes de algunos de estos miembros del jurado. Por ejemplo, de Hota y

"Le gustan los proyectos intermedios, pero a menudo se acuerda de su pasado y ayuda a valorar los proyectos modernos Casi soy de la opinión del vieio Ber pre, ave ha dicho que siente que el concurso no se haya hecho unos años más tarde. Ciertamente el movimiento moderno estarí entonces tan desarrollado que habrí sido inevitable una victoria. En el caso de muchos proyectos modernos reacción tendŕ el juego demasiado fácil a causa de una reacción tendria el juego

Esta carta, entre líneas, manifiesta muchas cosas interesantes. Por una parte, la variedad de temas indica un trabajo común cada vez mayor, aunque todavía quizà no íntimo, ya que Giedion se siente en la obligación de insistir en cómo es su militancia a favor de lo moderno. En todo caso, la implicación en el asunto de Ginebra es an importante que lleva a compartir opiniones y datos confidenciales sobre el jurado. En realidad, si miramos as fechas, estas revelaciones rozan la ilegalidad, porque todavía se estaba deliberando el 14 de abril y, de hecho, no se comunicó el veredicto hasta el 5 de mayo 22 . Aunque al principio Giedion elude mencionar sus fuentes ("estamos aquí-se puede imaginar por qué vías-") acaba

18. FLC E2(03) 96 . d'être répondus"

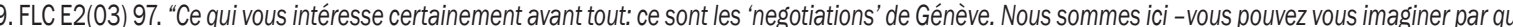

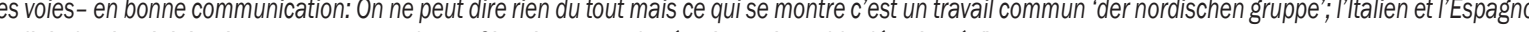

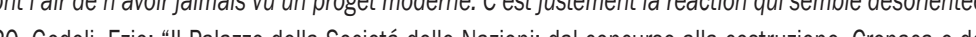

20. Godoli, Ezio: "Il Palazzo della Societá delle Nazioni: dal concurso alla costruzione. Cronaca e documenti" en Anzivino, Ciro; Godoli, Ezio: Ginevra 1927:

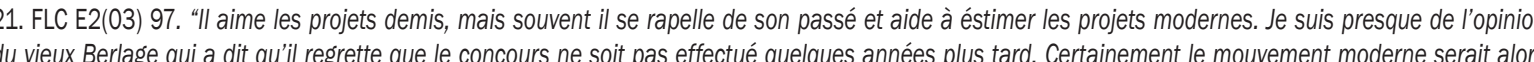
tellement developpé qu'une victoire aurait été inevitable. Chez beoucoup des projets modernes la reaction aurait le jeu beaucoup trop facile à cause d'ne

22. Quincerot, Richard: "Lec champ de Bataille du Palais des Nations 1923-31". En AA. W: Le Corbusierà Genève 1922-1932. Laussane: Payot, 1987, pp. 39y41. 
identificando como interlocutor a Moser, colega suyo en la universidad de Zurich. El empeño de Moser por beneficiar las propuestas de vanguardia debía ser grande cuando no ve problema en reflejar por escrito en una carta a Giedion sus impresiones sobre un concurso todavía no resuelto: "Moser me ha escrito desde Ginebra que está lleno de esperanza. Puede que yo haga, con un editor suizo, un pequeño opúsculo sobre los trabajos de la vanguardia en cena de los mejares proyectos. compromiso! Hay que pensar que los jueces son todos unos vejetes que no pueden tener la fuerza de luchar con el último encarnizamiento"z3.

El apoyo de Giedion fue activo, porque apenas un mes después pasa a la ofensiva en la opinión pública mediante una defensa ardiente en un diario zuriqués ${ }^{24} \mathrm{y}$, luego, en uno alemañn ${ }^{25}$ diez dias más tarde. Este empeno común dejo huella, porque en el capitulo de Giedion sobre Le Corbusier, en Espacio, Tiempo y Arquitectura26, se explaya mostrando la batalla por el concurso, que el interpreta en la práctica como victoria de la razón, cuya aplicación concreta dara lugar a las formas modemas como ocurifia en el falso techo de la Gran Sala, proyectado para el edificio de la Sociedad de Naciones:

"En la forma de solucionar el cielo raso, Le Corbusier, de una manera inconsciente, siguió el ejemplo de sus predecesores. Del mismo modo Davioud, despues de 1870, recurrio al cielo raso parabolico en un proyecto de tea-

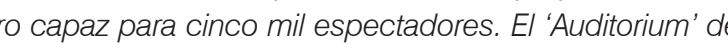
Adler y Sullivan, en Chicago (1887) -la más bella sala de

23. FLC E2(03) 97. “Moser m'a écrit de Génève quil lest plein d'espoir. Peut-être je ferai chez un éditeur Suisse un petti bouquin sur les traveaux de lavantGarde au concours de la SadN sont tous des vieillards qui ine peuvent avoir la force de lutter avec le dernier acharnement"

24. Giedion, Sigfried: Neue Zurcher Zeitung 15 de mayo de 1927.

25. Giedion, Sigfried: Frankiurter Zeitung 22 de mayo de 1927.

26. Giedion, Sigfried: Espacio, Tiempo y Arquitectura. Barcelona: Editorial Reverté, 2009. Edición original 1944.

27. Ibidem, p. 552.

28. Cartas de Le Corbusier a Loyd, el 9 y 12 de junio de 1927. Carta de Le Corbusier a Huston, el 13 de junio de 1927. Archivos de la SDN

29. Anzivino, Ciro; Godoli, Ezio: Ginevra 1927: il concorso per il Palazzo della Societá delle Nazionie il caso Le Corbusier. Fiorence: Modulo Editrice, 1979. p. 83. Esta posibilidad se apunta en una carta de Le Corbusier a Berlage, el 6 de junio de 1927.

30. Le Corbusier: Une maison - un palais. Paris: Editions G. Crès \& Cia, 1929.

31. Boesiger, W. Stonororv, 0. (Ed.): Le Corbusier et P Pierre Jeannerete, Oeuvre complète 1910-29 vol. 1. Zurich: Editions Girsberger, 1937 (edición en franncés) pp. 160 yss.

Uniones de su tiempo- está modelado siguiendo análogamente las exigencias de tipo acústico, y todavía en El propio Le Corbusier escribió en numerosas ocasioes a la Sociedad de Naciones y a miembros del jurado por ejemplo para enviar diseños complementarios, promar las ventajas de su concepción $n^{28}$. Hasta Gustave Lyon (1857-936), ingeniero acústico (suizo por cierto) en Cuuna carta de apoyo.

解 conocida del interés de Le Corrse con el proyecto, es su intento fina

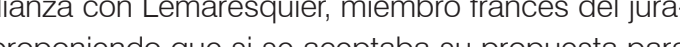
Palacio, la SDN tendría que nombrar a una, o mejor , personas de confianza para seguir la obra y que Lemaresquier ${ }^{2}$ y una temprana comprensión de la importancia es para conseguir que las ideas triunfaran. Ya en aque momento fue un gran divulgador que le dedicó una publicance, "La opinión pública se conmovió violentamente ante "la injusticia: la gran prensa internacional cotidiana, las cistas especializadas y las ideológicas debatieron la stión

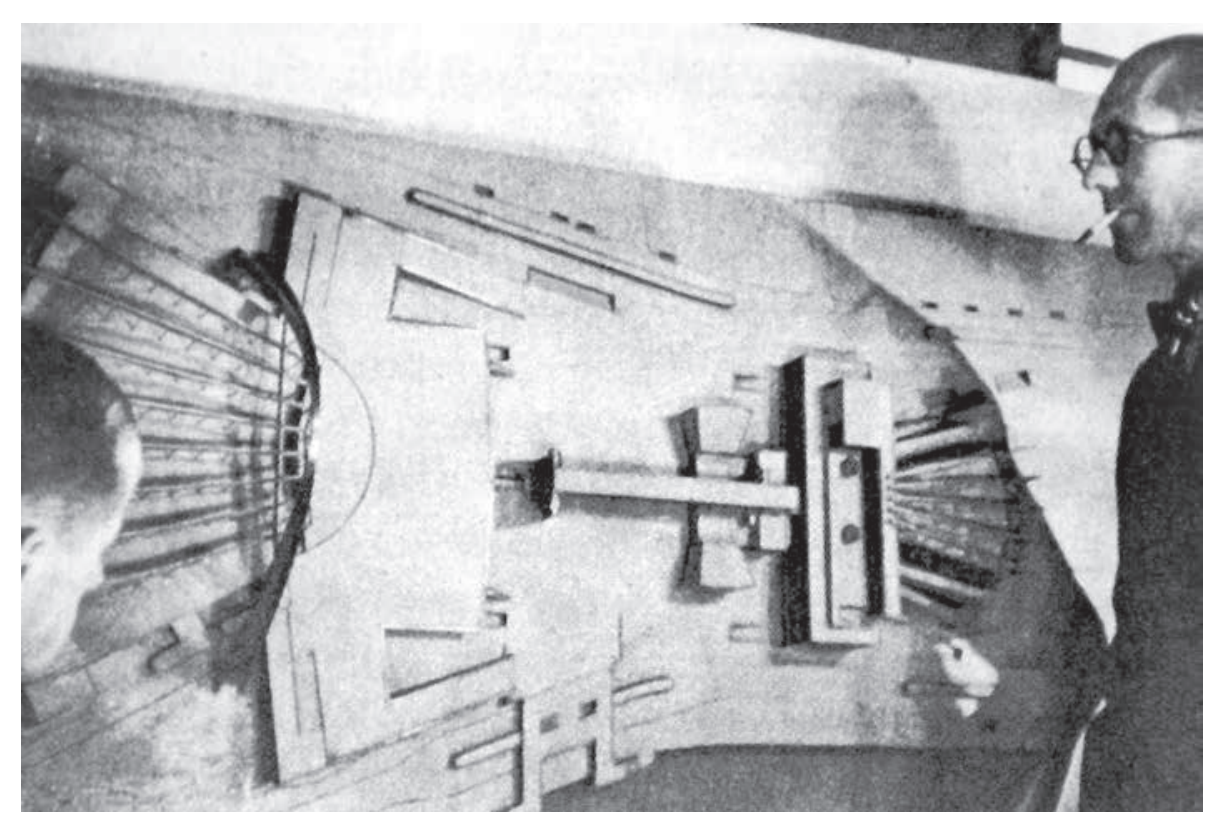

Además, estableció una interpretación ideológica de lo sucedido. Desde el primer momento insistió en que se estaba rechazando todo lo moderno precisamente por hecho de serlo:

Si este proyecto provoco tal impacto en opinión publica es porque representaba al espilitu moderno en lucha contra las rutinas y la Academia. (...) Este proyecto proponia en su momento un concepto estético nuevo acorde con la evolución general de la sociedad contemporánea" ${ }^{\prime 32}$

EL PALACIO DE LOS SOVIETS (1931-32)

Como es conocido, las diversas peripecias en torno Palacio de las Naciones duraron hasta 1931. Una vez que las esperanzas puestas en la nueva utopia del gobierno universal se dieron por perdidas, nuestro arquitecto decdió probar suerte en la URSS, que todavia era vista como una novedosa experiencia política, junto a otros regíme-

El Palacio de los Soviets era la oportunidad de construr marco para el nuevo hombre surgido de la revolución.

32. Ibidem

33. Las siguientes palabras sirven como ejemplo de su interés por el nazismo: "En esta severa conmoción, una chispa de bien: Hitter reclama materiales sannos y desea, con esta vuelta a las tradiciones, reencontrar l a robusta salud que se puede descubrir en toda raza, cualquiera que sea". Le Corbusier: Sur les quatre

35. FLC 12(05) 73.

36. FLC 12(05) 74

37. FLC 12(20) 80. 38. Según las tablas de conversión del valor del dólar, se puede estimar que 1 de 1931 , equivale a 14 \$ unos 10 € de 2012, así que los honorarios fijados
Aunque en la URSS de 1931 ya empezaban a tomar cuerpolas tendencias clasicistas y el realismo, se invito a tomar parte en el concurso a un grupo concreto de arquitectos os (igura 4). En esa selección quedaba de manifies-

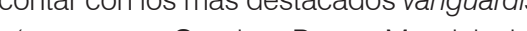
ence (entre otros Gropius, Perret, Mendelsohn, (to (lias que se rralaba de una nueva oportunidad, después del Conch este concurso restringido. Se le ofrecía, como a los demás, una cantidad de dos mil dólares y en su respuesta de fecha 10 de septiembre de $1933^{\circ 0}$ después de asegurar que la proposición le interesaba mucho desde

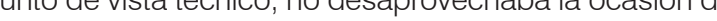
pecoldar que sus servicios no costantan menos de cuatro ce Al inal, sabemos que se fimó un contrato (1) de percibir tres mil dólares ${ }^{38}$. Le Corbusier, un idealista 
5. Croquis de Le Corbusier con las distrintas etap
Soviets. promotor de los nuevos tiempos, no dejaba de puntualizar los problemas más prosaicos y de hacer valer sus condiciones. A esas alturas, se veía claramente como un Peroladecepción llegóel 28 defebrero de 1932 cuando el jurado otorgó un premio al veterano arquitecto neopalladiano I.V. Zoltovskii, al joven representante de la corriente proletaria soviética B.M. Iofan y al americano H.O. Hamilon $^{39}$. Comenzaba aquí un proceso de puesta en marcha del proyecto ganador que culminaría en 1934, con la transormación del proyecto inicial de Zoltovskij en una especie de enorme mausoleo con forma de pastel de bodas Le Corbusier, unavez conocido el fallo inicial del jurado no se quedo callado y movilizo a todos los conocidos para conseguir que se reconociera la calidad de su propuesta. Nosinteresaenespecial surelaciónconNikolaj Colly (1894(966) ${ }^{40}$, que habia trabajado para Le Corbusier como coordinador del grupo de técnicos que adecuo el proyecto de Centrosoyus (1928-1930) a la normativa y a las peculiardades sovieticas. Le Corbusier le envio dos cartas, una el 6 de abril de 1932, que se extravió y otra el 15 de abril del mismo año. En esta untima se quejaba de las pocas condiciones del premiado, alababa la solución que el aporto y, en el fondo, pedía ayuda y comprensión a su contacto Pero he aquilo importante: No se puede dejar que el Palacio de los S. se haga en las condiciones actualmente señaladas a la opinión. Recibo noticias de America. El premiado es un hombre joven del todo, sin gran preparación

profesional. Dice que no ha hecho una sección para su proyecto i'porque el programa no lo pedía'! (...) Nosotros hemos aportado a Moscú, con nuestro envio, la colaboacion apasionada de gente de experiencia y de talento. como Gustave Lyon. (...) Este es un gran momento para Rusia. ¿Cual es pues la opinion en todo esto de los grupos .

El buen colaborador respondio dando la razón a su amigo en carta del 25 de abril de 193242, disculpándose por no haberlo hecho antes, ya que formaba parte de conite que decidia la solución definitiva del Palacio de se Sovicts. Esto demuestra que la lucha por un premio no se limitó al diseño e inclúa, de nuevo, apoyos dentro de mismo jurado, que incluso proporcionaron información sobre las deliberaciones. La historia de Ginebra se repite. Se vuelve a observar una red internacional de contactos, desde la URSS hasta USA. Además, la identificación de (aborador con Le Corbusier era elevada, hasta el punto de referise a las causas de la derrota con la conocida . "Usted conoce, ahora, la opinion y la decision oficial. Todavia hay ojos que no ven. No se ha querido comprender uproyecto, se buscaba, en vano, una nueva monumentaIdad, digna de la epoca, ly no se ha captado que esta se

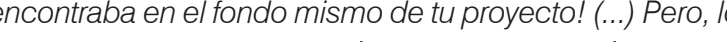
repito, por el momento ihabla ojos que no querian ver! "h $^{2}$ Las reacciones de olros componentes del MovimienModerno no se dejaron esperar. Inmediatamente se

39. Ver a este respecto el capitiulu “La "rrahison dramatique' du Palais des Soviets" en el libro: Cohen, Jean-Louis: Le Corbusier et la mystique de r'URSS, (20xelles-Liège: Piere Mardaga éditeur, 1987, pp. 205-2

40. En sus cartas, Le Corbusier transcribe el apellido como Colly y por eso lo mantenemos así. En el libro de Jean-Louis Cohen se ha usado Kolli. En esta obra

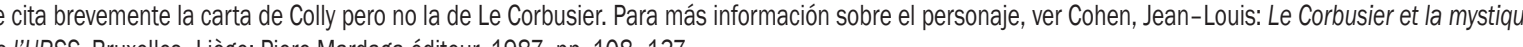

政

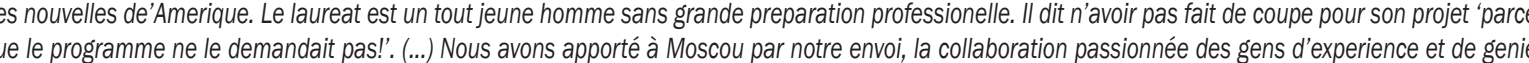
comme Gustave Lyon. (...) C'est pour la Russie un grand moment. Quelle est donc l'opinion dans tout ça des groupes de nos camarades? Ecrivez le moi s.v.p."

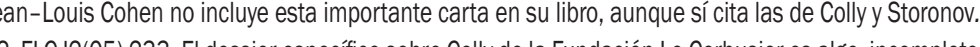

(11-1933, la FLC

43. Le Corbusier: Vers une architecture. Paris: Editions G. Crès \& Cia, 1923. Reedición de 1958. p. 64.

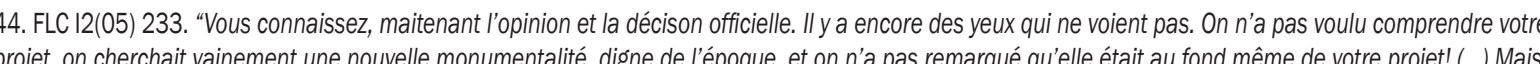

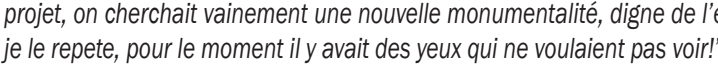

formó un frente común contra el fallo del jurado, que no dudó en recurrir al propio Stalin en defensa de la nueva arquitectura. Le Corbusier no olvido esta decepción y un vez más la convirtió en emblema de su lucha. En Vers une architecture, en las notas a la reedición de 1958 incluye una fotografia antigua de la maquela cuyo tex lo dice: 1931. Palacio de los Soviets. (Rechazado)

Una de las primeras cartas de apoyo fue aquell mencionada por Le Corbusier cuando decía a Colly: "recibo noticias de America". Desde Estados Unidos Alfred Kastner y Oscar G. Stonorov, arquitectos, se diigen a Le Corbusier en carta del 22 de marzo de 1932 manifestando al maestro su repulsa hacia el proyecto ganador del concurso del Palacio de los Soviets en el que ellos habian obtenido el segundo premio. El alemán Oscar Stonorov (1905-1970) habia sido, junto a Willy Boesiger el editor del primer tomo de la Obra Completa en alemán, en $192^{\circ}$. Ya en USA, adonde emigró en 1029, le envaba folos de su proyecto y quería conocer las ideas da Le Cobusier Hacla causa común contra la acadenia y, en un tonojuvenlly exallado, se quejabade

45. Le Corbusier. Vers une architecture. Paris: Editions G. Crès \& Cia, 1923. Prefacio.

46. La referencia de esa edicion en alemán es Boesiger Willy; Stonorov Oscar. (Ed):) Le Corbusier \& Pierre Jeanneret ihr Werk von 1910-1929. Zurich: Les 20 En este articulo citaremos la edición en francés de 1937, coordinada por los mismos autores y de la misma editorial. que le programme ne demandadit pas une section le e uualifie parmi les héros des Beaux Ant Russes sont-ils si gelés que le reste des occidentaun"!

Respecto a la focalización de todas las críticas con-
2

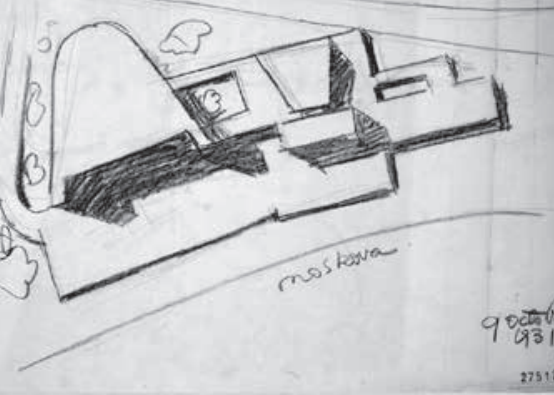

.... Parece que M. Albert Kahn, dios-ingenieroyc ru . Su M. Hamiton) explicación, que el programa no pedín

Se añadió una posdata con fecha 6 de abril, en la Encian:

Entre tanto, Mr. Hamilton ha sido nombrado arquitecto en jefe de la construcción del Palacio. Es inaudito..... ¿Es que ra historia se replie? iLos rusos estan tan bloqueados tra el academicismo, es interesante comprobar que la distancia es más ideológica que conceptual, porque un vistazo a los esquemas en planta de los dos concursos quent ésétodo de composición por partes belondo siaprendido de contione de Duand Guadet (igura 5). En la caja que sobre con réńn centan las distintas estancias que pedí el prog repre- y sentan las distintas estancias que pedia el programa, y bió el proyecto medeste los pimeros pasos se concimediante la yuxtaposición de fragmentos 


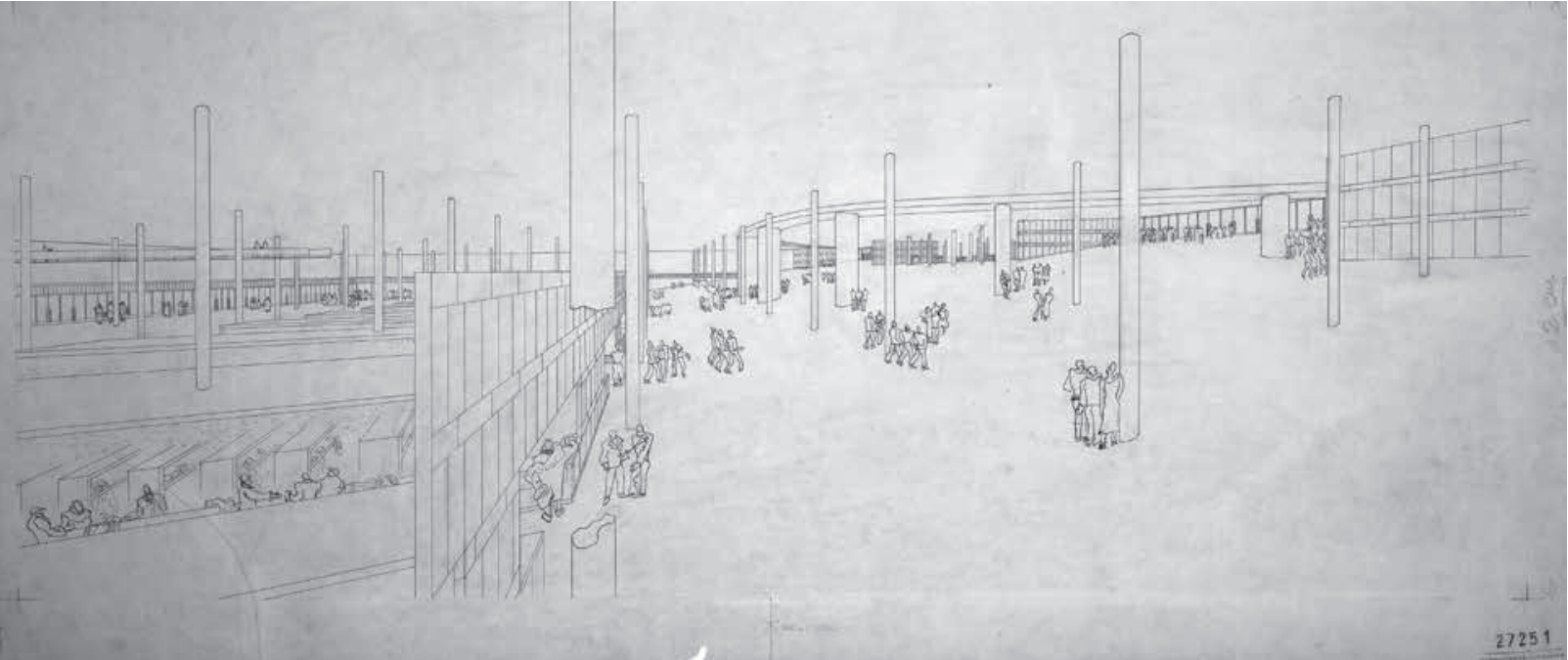

autónomos en sí mismos ${ }^{49}$. Lo que destaca en todas las calcas er pondiciones acústices la perticipación delingeniero suizo Gustav Lyon, en auyas teoŕs se fundamentó la propuestade Ginebra y a que ahora se contrató expresamente para encourso que ahora secontrato

Estos con miembros de Movimiento Moderno, buscando el apoyo para su procarácter más formal a través de los CIM y su bra ejectivo el CIRPAC, organizaciones a cuya fomacín contribuyó el fiasco del concurso de Ginebra Son los CIAM quienes se responsabilizaron de la defensa de Le Corbusier con los dos conocidos mensajes al propio Le In, firmados por el presidente de los congresos Cornelis an Eesteren y el propio Sigried Giedion

dencia entre ellos, en abril de 1932, es Giedion quien articuló toda la operación, con Le Corbusier siempre al tanto. Le Corbusier

envió a Giedion una carta pidiendo informaciones varias 16 de abril de $1932^{20}$ en la que volvía a quejarse de vera una seccín porque no la pedí de Hranton no tubién insistí en la poca capacitacín profesional de este oven americano Conclúa diciendo en tono apasionado: "Il faut luterl" " "ihay que lucharl"). La respuesta de Gie dion a este grito de combate no se hizo esperary to fo cha de 23 de abril. En esa carta le envió el primer escrito Dor los CIAM y el CIRPAC, que teńa ono de un manifiesto.

No se había designado ningún jurado, pero aparea ceredicto que se daría, estaría al y que reprear a que conduce el plan quinquepensamiento contemporáneo. El Palacio de los Soviets, habla el lenguaje infalsificable de la arquitectura Explicaría la revolución alcanzada por la civilización nueva de los tiempos modernos" 51 .

\section{FLC 1205)}

51. FLC 12(05) 236. “...Aucun jury n'était designé mais il a pparissait, comme évident que le verdict qui serait porté, serait inspiré de la ligine génerale qui conduit le plan quinquenal et representerait la plus éclatante manifiestation de la pensée contemporaine. Le Palais des Soviets, parle le langagé infalsifiable de 'architecture. Exprimerait la revolution acomplie par la civilisation neuve des temps modernes".
Le Corbusier respondió a Giedion el 25 de abril de Pero... repentinamente: ila reacción violenta! El Palacio 193252. haciendo comentarios sobre el escrito de los de los Soviets, coronamiento del Plan Quinquenal, sería CIAM a Stalin (figura 6). El tono entre ellos cada vez era en estilo Renacimiento italiano..."5r.

más coloquial, lejos ya de la seriedad de los comienzos de su relación en la epoca de 1925. Le Corbusier empezaba con un "Mon cher Giedion" para continuar bromeando sobre el reciente viaje de este a Barcelona:

"¿Se ha dejado llevar por una andaluza? En el CIRPAC enía usted el aspecto de un hombre agrietado y yo el de un diletante. La realidad es que es usted un disfrutón y yo un trabajador"s3.

Se quejaba de que esta carta no tuviera mayúsculas al comienzo de frase ni en los nombres propios. Enseguida, el 28 de abril de $1932^{54}$, Giedion volvió a envia noticias a Le Corbusier sobre varios temas, entre ellos el nuevo escrito que habian remitido a Stalin ${ }^{55}$. Aprovechaba para matizar que la primera carta que recibió Stalin sí iba con mayusculas, pues el CIRPAC también es clásico, y que solamente la copla que recibió Le Cor busier, escrita en el despacho de Steiger, iba puntuada tan extrañamente. Llama la atención que en medio de ema principal, Le Corbusier tenga liempo para puntualizar cuestiones sobre tipografía. Su comentario se entiende mejor si se tienen en cuenta las propuestas de la Bauhaus en 1925 para suprimir el uso de mayúsculas en aras de la simplicidad ${ }^{56}$, algo que el rótulo de su propia sede contradecía.

Un buen resumen de todas las incidencias que hemo comentado, pueden ser estas palabras de la Obra Completa, similares a las usadas para el Palacio de las Naciones. "Este proyecto no tuvo suerte. Despertó interés considerable en Moscú. Se tomó en cuenta la ejecución.

\section{EL CONCURSO COMO VEHICULO DE LA} MODERNIDAD

Este recorrido por las reacciones de diversos colegas encos concursos clave de la historia arquitectónica moderna permite sacar varias conclusiones sobre algunas categorias propias de la modernidad ${ }^{50}$. De una parte se aprecia el tono dialectico, por el que toda vanguardia, necesita un oponente frente al que singularizarse y al que combatir heroicamente. Aquí se materializa en la "réaction" academicista.

También destaca la crecionte internacionalización de las relaciones, que desembocó en organismos de encuentro y difusion como los CIAM. En lo que respecta Le Corbusier, salió de estas batallas con su posicion de figura predominante muy reforzada. Un pequeno indicador de ese aumento de proyección, es que entre 1920 y 1929 solo se conservan en sus archivos siete peticiones de información ${ }^{59}$ de gente poco conocida, mientras que en la década siguiente aumentan hasta cincuenta y ocho ${ }^{60}$ inchen carlas de instiuciones de todo el planela como Mitsubishi S.A., Guggenheim, el Gobierno de Australia o la Architectural Association.

Por último, se observa que las nuevas propuestas poco éxito a juzgar por los resultados. En cambio, fue más fructirera la incorporación al discurso teorico y forma analizada se revisten las reacciones con argumentos

52. FLC 12(05) 237

53. FLC 1205) 237. “Vous vous étes fait enlever par une Andalouse? A CIRPAC vous aviez l'air d'un homme craqué, et moi d'un dilettante. La realitié, est que vous êtes un jouisseur et moi un travailleur".

54. FLC 12(05) 238

56. Herbert Bayer, profesor de tipografía en la Bauhaus entre 1925 y 1928 decia sobre esto: "¿por qué doble cantidad de signos, si se logra lo mismo con Ia mitad?" Satué, Enric: El diseño grataico. Desde los origenes hasta nuestros dias. Madrid: Alianza Forma, 1992. p. 135.

57. Boesiger, W.; Girsberger, H. (Ed.).:Le Corbusier et Pierre Jeanneret, Oeuvre complete 1929-34 vol. 2. Zurich: Editions Girsberger, 1934, pp. 123 y 124 58. Otxotorena, Juan Miguel: Arquutectura y proyecto moderno. Barcelona: Editorial Eunsa, 199.

59. FLC T1(4) 320-327.

60. FLC T1(4) 328-386. 
cientificos, como corresponde a una modernidad funcional inspirada en el método ilustrado. Se insiste en la necesidad de entender el proyecto desde la sección, o se vincula la forma de las salas a cuestiones acústicas. Se destaca el valor de contar con ingenieros dentro del equipo y se argumenta

En definitiva, estas cartas personales, aunque coinciden con el tono de la correspondencia oficial, permiten vislumbrar desde dentro la polemica y las relaciones que acompañaron a estos dos concursos. Quizás la mejor confirmación de ese ambiente de ruptura sean las pala政 ibro y manifilesto que, por cierto, leyo cuando todavia era un borrador en la Residencia de Estudiantes de Madrid en su viaje a nuestro país de 1928

Que lejos estamos de las Academias' Estamos en el te la responsabilidad. iQué lejos del Pre-

Fernando Zaparaín Hernández (Burgos 1964) arquitecto (1989) y doctor (1995) por la Escuela de Arquitectura de Valladolid,

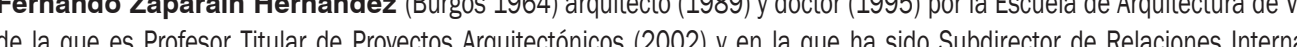
(2002-2008). Autor de los libros Le Corbusier, artista-héroe y hombre-tipo (1997). Arquitectura para el siǵglo XXI en Castilla y Leán (2011) y Cruces de caminos. Álbumes ilustrados: construcción y lectura (2010), premio a la mejor monografía de Ciencias Huma nas y Sociales de Edición Universitaria (2011). Ha publicado en las revistas Arquitectura Viva, EGA, Ra y Minerva. Es investigador en proyectos de I+D+i de la Junta de Castilla y León y del Ministerio de Ciencia e Innovación. Sus edificios dotacionales como el Centro Aspaym (medalla de Plata en la Trienal Interarch 1997), la Escuela Deportiva Niara (2010) o la bodega Carraovejas (2012), se han

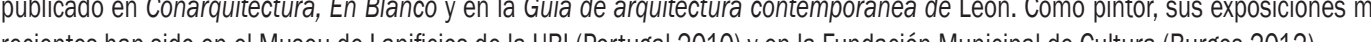




\section{Autor imagen y fuente bibliográfica de procedencia}

Información facilitada por los autores de los artículos: página 20, 1 (AA.W.: Concurso de Proyectos 1996. Propuestas de los equipos adjudicatarios. Sevilla: Dirección General de Arquitectura y Vivienda, Junta Andalucía, 1997, portada), 2 (Dirección General de Arquitectura y Vivienda, Junta Andalucía, tríptico); página 22, 3 (Manuel Toledo), 4 (Andrés López); página 23, 5 y 6 (Jesús Granada) 24, 7 (Fernando Alada), 8 (Jesús Granada), 9 (Fernando Alda); páginas 42, 43 y 44, 1 a 3 (El Croquis n53 0MA/Rem Koolhaas. Madrid: El Croquis, 1994, pp. 73 y 78); páginas 46, 47, 4 a 6 (AA.VV: Exposición Universal Sevilla 1992: ideas para una ordenación del recinto, Sevilla: Expo 92, Comisaría General de España, 1986); página 49, 7 (Ceccarelli, P. La construcción de la ciudad soviética. Barcelona: Gustavo Gili, S.A., 1972, figura 26, Anexo ilustraciones, p. XI), 8 (De Michelis, M.; Pasini, E. La Citta`Sovietica 1925-1937, Venecia: Marsilio Editori, 1976, p. 80); páginas 50y 51, 9 y 10 (9. De Michelis, M.; Pasini, E. La Citta 'Sovietica 1925-1937, Venecia: Marsilio Editori, 1976, pp. 81y 180); página 55,1 (Reproducido de Deutches Dokumentation fur Kunstgeschichte, Universitat Marburg, en www.fotomarburg.de); página 56, 2 (Cristina Gastón Guirao); página 57, 3 (Der Scherei nach den Turmhaus. Der IdeenwettbewerbHochhaus am Bahnhof Friedrichstrasse. Berlin 1921/22. Berlín: Argon Verlag GmbH, 1988, p. 39), 4 (Cristina Gastón Guirao en base a Der Scherei nach den Turmhaus. Der IdeenwettbewerbHochhaus am Bahnhof Friedrichstrasse. Berlin 1921/22. Berlín: Argon Verlag GmbH, 1988, p. 38); página 58, 5 (AAVV. Mies in Berlin. (publicado en relación con la exposición del mismo nombre). New York/Berlín: MOMA, 2001, p. 183); página 59, 6 (Reuter, Helmut; Schulte, Birgit. Mies and modern living. Interiors, furniture y photography. Ostfildern: Hatje Canz Verlag, 2008, p. 50, 210 y AAVV. Mies in Berlin. (publicado en relación con la exposición del mismo nombre). New York/Berlín: MOMA, 2001, p.181), 7 (AAVV. Mies in Berlin. (publicado en relación con la exposición del mismo nombre). New York/Berlín: MOMA, 2001, p. 182); página 60, 8 (Cristina Gastón Guirao en base a Der Scherei nach den Turmhaus. Der IdeenwettbewerbHochhaus am Bahnhof Friedrichstrasse. Berlin 1921/22. Berlin: Argon Verlag GmbH, 1988, pp. 45, 105); página 62, 9 y 10 (Reuter, Helmut; Schulte, Birgit. Mies and modern living. Interiors, furniture y photography. Ostfildern: Hatje Canz Verlag, 2008, pp. 230, 252, 258); página 64, 11 (Zevi, Bruno (ed): Erich Mendelsohn. Opera Completa. Turín: Testo \& Immagine, 1997, p. 197), 12 (Der Scherei nach den Turmhaus. Der IdeenwettbewerbHochhaus am Bahnhof Friedrichstrasse. Berlin 1921/22. Berlín: Argon Verlag GmbH, 1988, pp. 181, 184); página 65, 13 (Schulze, Franz. Mies van der Rohe. Una biografía crítica. Madrid: Hermann Blume, 1986 p. 155); página 69, 1 (Inv. Nr. HP 041,005. Architekturmuseum der Technischen Universität Berlin in der Universitätsbibliothek); páginas 70, 72, 73, 74, 2 a 5 (@FLC/Vegap, Sevilla, 2012. @Pierre Jeanneret, Vegap, Sevilla, 2012), 6 (Theaterwissenschaftliche Sammlung Universität zu Köln); página 75, 7 y 8 (Inv. Nr. 2750, Inv. Nr. F 1604. Architekturmuseum der Technischen Universität Berlin in der Universitätsbibliothek); páginas 76 y 80, 9 a 11 (@FLC/Vegap, Sevilla, 2012. @Alexander Rodchenko, Vegap, Sevilla, 2012), 12 (0148126. Tretyakov State Gallery, Moscow); página 82, 13 (Austrian Frederick and Lillian Kiesler Private Foundation Vienna), 14 ((СFLC/Vegap, Sevilla, 2012. @Pierre Jeanneret, Vegap, Sevilla, 2012); página 84, 15 (@FLC/Vegap, Sevilla, 2012. @Pierre Jeanneret, Vegap, Sevilla, 2012), 16 (X1119.3a-c. Colletion of Brooklyn Museum, New York); página, 85, 17 (Cabinet Ejzenstejn, Moscow (Hayuнoмемориальный кабинет-музей С. М. Эйзенштейна), 18 (Imagen de dominio público. http://commons.wikimedia.org/wiki/File:Sadanji_Ichikawa_Il_and_Sergei_Eisenstein. jpg); páginas 92 y 94, 1 y 2 (Roth, Alfred: La nouvelle architecture presentée en 20 exemples, Die neue architektur dargestellt an 20 Beispielen, The new architecture presented in 20 examples. 1930-1940. Zúrich: Verlag für Architektur Artemis, 1975, pp. 131, 132); página 94, 3 (Architettura, № XVI, Fasc. IX. Milán: septiembre de 1938, p. 574); página 96, 4 (Roth, Alfred: La nouvelle architecture presentée en 20 exemples, Die neue architektur dargestellt an 20 Beispielen, The new architecture presented in 20 examples. 1930-1940. Zúrich: Verlag für Architektur Artemis, 1975, p. 136), 5 (Fondazione Giuseppe Terragni; reproducida en Curtis, William J. R.: La arquitectura moderna desde 1900. Londres: Phaidon, 2006, p. 366); página 97, 6 (International Institute of Social History, Amsterdam; reproducida en Molema, Jan: Jan Duiker. Barcelona: Gustavo Gili, 1996, p. 85.), 7 (Emilio Cachorro Fernández); página 98, 8 y 10 (Roth, Alfred: La nouvelle architecture presentée en 20 exemples, Die neue architektur dargestellt an 20 Beispielen, The new architecture presented in 20 examples. 1930-1940. Zúrich: Verlag für Architektur Artemis, 1975, p. 137), 9 (AA.W.: Iǵnazio Gardella, 1905-1999. Arquitectura a través de un siǵlo. Catálogo de la exposición. Madrid: Ministerio de Fomento, 1999, p. 13); página 100, 11 y 12; página 102, 13 (Maffioletti, Serena: BBPR arquitectura. Banfi, Belgiojoso, Peressutti, Rogers. Sevilla: COAAO, 1996, pp. 47, 56, 67), 14 (Musée du Petit Palais, Ginebra; reproducida en Stoichita, Victor l.: Ver y no ver. Madrid: Siruela, 2005, p. 38); página 108, 1 (Arquitectura. Agosto 1969, № 128. Madrid: Colegio Oficial de Arquitectos de Madrid, 1969. Primera página; Nueva forma: arquitectura, urbanismo, diseño, ambientes, arte. "Concurso Universidad Autónoma de Madrid". Septiembre 1969. № 44. Madrid, 1969. Portada; Nueva forma: arquitectura, urbanismo, diseño, ambientes, arte. "Concurso Universidades Autónomas de Bilbao y Barcelona". Enero 1970. № 48. Madrid, 1970. Portada); página 110, 2 (Nueva forma. Septiembre 1969. № 44. cit. p. 5); página 111, 3 y 4 (Arquitectura. Agosto 1969, № 128. cit. pp. 33 y 35); páginas 112 y 113, 5 a 9 (Nueva forma. Septiembre 1969. № 44. cit. pp. 33, 30, 81, 18, 24); páginas 114, 115 y 116, 10 a 14 (Nueva forma. Enero 1970. № 48. cit. pp. 23, 20, 56, 69, 96), 15 y 16 (Cuadernos de arquitectura. Primer trimestre 1970. № 75. Barcelona: Colegio Oficial de Arquitectos de Catalunya, 1970. pp. 58 y 66); página 117, 17 (Nueva forma. Enero 1970. № 48. cit. p. 31); página 118, 18 (Nueva forma. Septiembre $1969 . N^{\circ} 44$. cit. p. 4; Temas de arquitectura y urbanismo. Septiembre 1969. № 123. Madrid-Barcelona, 1969. p. 5); página 123, 1 y 2 (Bucci, Ângelo; Puntoni, Álvaro; Vilela, José Oswaldo. Foto: Nelson Kon.); página 126, 3 (Parada, Sérgio Roberto. Projeto cedido por Rodrigo Biavati), 4 (Gorgati, Vinicius; Franco, Fernando de Mello; Moreira, Marta; Braga, Milton. Projeto cedido pelos autores), 5 (Rodrigues, Sidney Meleiros; Saraiva, Pedro Paulo de Melo; Rosemberg, Marcelo; Vaisman, Jacobina; Lobo, Marcos Toledo; Nunes, Ronaldo Soares.Projeto cedido pelos autores); páginas 128, 129, 6 a 12 (Bucci, Ângelo; Puntoni, Álvaro; Vilela, José Oswaldo. Projeto cedido pelos autores); página 131, 13 y 14 (Bucci, Ângelo; Puntoni, Álvaro; Vilela, José Oswaldo. Foto: Nelson Kon); página 136, 1 (Fromonot, Françoise. En Jørn Utzon, architetto della Sydney Opera House. Milano: Electa, Documenti di Architettura, 1998. pp. 15), 2 (Piano, Renzo. En Giornale di Bordo. Florencia: Passigli Editori, 1997, pag 38); página 138, 3 (Mikami, Yuzo. En Utzon's sphere. Sydney Opera House. How it was designed and built.Tokio: Ed. Shoku Kusha, 2001), 4 (Silver, Nathan, The making of Beuabourg, A building biography of the Centre Pomidou, Paris. Cambridge, Massachussets: Ed The MIT press, 1994); página 139, 5 (Piano, Renzo, Renzo Piano Building Workshop, complete works. Londres: Phaidon Press Limited, 1993. vol 1. pp. 54), 6 (Weston, Richard: Utzon : inspiration, vision, architecture. Hellerup (Dinamarca): Editorial Blondal, , 2002. pp. 117); página 140, 7 (Weston, Richard: Utzon: inspiration, vision, architecture. Hellerup (Dinamarca): Editorial Blondal, , 2002. pp 116), 8 (Fromonot, Françoise. En Jørn Utzon, architetto della Sydney Opera House. Milano: Electa, Documenti di Architettura, 1998. pp 64), 9 (Ferrer, Jaime, Jørn Utzon. Obras y proyectos. Barcelona: Editorial. Gustavo Gili, 2008. pp. 159); página 10, (Rice, Peter: "La structure métallique". En Architecture d'aujourd'hui. Febrero 1982, n 219. pp. 62. París: Editorial Groupe Expansion,1930), 11 (Piano, Renzo, Renzo Piano Building Workshop, complete works. Londres: Phaidon Press Limited, 1993. vol 1. pp. 45); página 142, 12 (Architectural Design', vol 47 nº 2, febrero 1977); página 144, 13 (Weston, Richard: Utzon : inspiration, vision, architecture. Hellerup (Dinamarca): Editorial Blondal, 2002); página 148, 1 (AA.W.: Arquitectura Viva. "Obras singulares". 1998, № 62. Madrid: Arquitectura Viva. 1998. pp. 78-81); página 149, 2 y 3 (AA.W.: Concurso 2G competition: Parque de la Laguna de Venecia = Venice Laggon Park. Barcelona: Gustavo Gill, 2008. pp. 81, 13); página 150, 4 (Jacopo de 'Barbari, [Web en linea]. [Consulta: 04-10-2012]), 5 (Zumthor, Peter: Atmósferas: entornos arquitectónicos, las cosas a mi alrededor. Barcelona: Gustavo Gili, 2006. p. 6); página 151, 6 (David Chipperfield 2006-2009. El Croquis. № 120. Madrid: El Croquis. 2008. p. 66); páginas 152, 153,7 a 9 (AA.W.: Carlos Ferrater. Works and Projects 1980-2000. Barcelona: Actar, 1998); páginas 154, 155, 10 a 12 (Enric Miralles + Benedetta Tagliabue: 1995-2000. El Croquis. № 72. Madrid: El Croquis. 2000. pp. 132-141); página 156, 13 a 15 (David Chipperfield 2006-2009. El Croquis. № 120. Madrid: El Croquis. 2008. pp. 64, 67, 66); página 157, 16,17 (Luca Nicolao Photography, [Web en linea]. [Consulta: 19-9-2012]), 18 (David Chipperfield 2006-2009. El Croquis. № 120. Madrid: El Croquis. 2006. p. 66); páginas 162, 164, 167, 169, 170, 1 a 6 (๑ F.L.C. /VEGAP, Sevilla, 2012. OPierre Jeanneret, VEGAP, Sevilla, 2012); página 176, 1 (García Mercadal y S. Giedion. Congresos internacionales de arquitectura moderna. AC. №5. Primer Trimestre de 1935. Barcelona. GATEPAC.1932 p.12); páginas 178, 179, 2 a 4 (AC. №11. Tercer trimestre de 1933. Barcelona. GATEPAC. 1933. pp. 37, 34, 35); página 180, 5 (Le Corbusier et Pierre Jeanneret : Ouvre Complète 1910-1929. Zurich : Les Édtions D’Architecture. 1964 p.65), 6 (AC. NN11. Tercer trimestre de 1933. Barcelona. GATEPAC. 1933. p. 34), 7 (Le Corbusier et Pierre Jeanneret: Ouvre Complète 1910-1929. Zurich : Les Édtions D’Architecture. 1964 p.65), 8 (L'Habitation minimun. Frankfurt. 1929. Zaragoza: Edición facsimil Colegio de Arquitectos de Aragón.1997.p.26), páginas 181, 182 (AC. №11. Tercer trimestre de 1933. Barcelona. GATEPAC. 1933. p. 37, 1); páginas 189, 190, 1 y 2 (Cássia de Souza Mota); página 191, 3 (Boero, Dolores; Castellote, Ana Maria; Puglisi, Jose Agustin. Fonte: http://www.uia-architectes.org/texte/ england/Celebration/SPrix/Reglll/Frames.html), 4 (Fernandez, Alberto; Guerrero, Camilo; Javiera, Paulina; Sanchez Recio, José Manuel. Fonte: http://www.celebcities2.org/); página 192, 5 y 6 (Nikita, Sergienko. Fonte: http://www.celebcities3.org/wc/content/18/pdf/054FECC2-C9DC-410A-A044-CB2A0BBD25EB.pdf) 